\title{
Acute Myeloid Leukemia with FLT3/ITD Mutation
}

National Cancer Institute

\section{Source}

National Cancer Institute. Acute Myeloid Leukemia with FLT3/ITD Mutation. NCI

Thesaurus. Code C126748.

Acute myeloid leukemia characterized by the presence of a FLT3-internal tandem duplication (ITD) mutation. Patients with this mutation usually present with more aggressive disease and have higher rates of relapse after remission. 